\title{
Preoperative miRNA-208a as a Predictor of Postoperative Complications in Children with Congenital Heart Disease Undergoing Heart Surgery
}

\author{
Keren Zloto $^{1}$ - Tal Tirosh-Wagner ${ }^{2}$ - Yoav Bolkier ${ }^{2}$ - Omer Bar-Yosef ${ }^{1} \cdot$ Amir Vardi $^{3}$ • David Mishali ${ }^{4} \cdot$ Gidi Paret $^{1}$. \\ Yael Nevo-Caspi ${ }^{1}$
}

Received: 17 July 2019 / Accepted: 1 October 2019 / Published online: 15 November 2019

(C) Springer Science+Business Media, LLC, part of Springer Nature 2019

\begin{abstract}
Major perioperative cardiovascular events are important causes of morbidity in pediatric patients with congenital heart disease who undergo reparative surgery. Current preoperative clinical risk assessment strategies have poor accuracy for identifying patients who will sustain adverse events following heart surgery. There is an ongoing need to integrate clinical variables with novel technology and biomarkers to accurately predict outcome following pediatric heart surgery. We tested whether preoperative levels of miRNAs-208a can serve as such a biomarker. Serum samples were obtained from pediatric patients immediately before heart surgery. MiRNA-208a was quantified by RQ-PCR. Correlations between the patient's clinical variables and miRNA levels were tested. Lower levels of preoperative miRNA-208a correlated with and could predict the appearance of postoperative cardiac and inflammatory complications. MiRNA-208a may serve as a biomarker for the prediction of patients who are at risk to develop complications following surgery for the repair of congenital heart defects.
\end{abstract}

Keywords Biomarker $\cdot$ miRNA $\cdot$ Congenital heart disease ·

Pediatric cardiac surgery $\cdot$ Prediction of complications .

Preconditioning

\section{Introduction}

Major perioperative cardiovascular events, such as cardiac arrest, arrhythmia, and pericardial tamponade, are important causes of morbidity in patients undergoing repair of

Gidi Paret and Yael Nevo-Caspi contributed equally to this work.

The work was performed at the Department of Pediatric Critical Care Medicine, Safra Children's Hospital, Sheba Medical Center, Tel Hashomer, Israel

Associate Editor Junjie Xiao oversaw the review of this article

Yael Nevo-Caspi

yael.caspi@sheba.health.gov.il

1 Department of Pediatric Critical Care Medicine, Safra Children's Hospital, Sheba Medical Center, Sackler Faculty of Medicine, Tel-Aviv University, Tel-Aviv, Israel

2 Department of Pediatric Cardiology, Safra Children's Hospital, Sheba Medical Center, Sackler Faculty of Medicine, Tel-Aviv University, Tel-Aviv, Israel congenital heart disease (CHD) [1]. A number of clinical risk indices have been developed, but their predictive power is limited [2], raising the need for a simple and strongly predictive noninvasive alternative. MiRNAs are a class of noncoding RNAs of approximately 22 nucleotides in length that
3 Department of Pediatric Cardiac Intensive Care, Safra Children's Hospital, Sheba Medical Center, Sackler Faculty of Medicine, Tel-Aviv University, Tel-Aviv, Israel

4 Department of Pediatric Cardiac Surgery, Safra Children's Hospital, Sheba Medical Center, Sackler Faculty of Medicine, Tel-Aviv University, Tel-Aviv, Israel 
was shown to be useful biomarkers in various diseases. The posttranslational regulatory roles of miRNAs have been demonstrated in almost all physiological processes. Unlike other extracellular RNA molecules, extracellular miRNAs are remarkably stable in serum and, together with their tissue specificity and the ease by which they can be detected and quantified, they have sparked great interest in their use as clinical biomarkers for a wide range of medical states [3]. We had reported that the level of circulating cardiac miRNA-208a following surgery can serve as a sensitive biomarker for the postoperative course of pediatric patients with CHD undergoing heart surgery for the repair of their defect [4]. In the present study, we hypothesized that preoperative miRNA-208a levels in the blood of pediatric patients with CHD can predict postoperative complications.

\section{Materials and Methods}

\section{Patient, Medical, Laboratory, and Surgical Parameters}

All pediatric patients with CHD who underwent cardiac surgery at Safra Children's Hospital between 2012 and 2016 and whose legal representative provided informed consent were enrolled. The inclusion criteria were age younger than 21 years and an echocardiographic diagnosis of CHD that required cardiac surgery. The laboratory parameters assessed in the study were as follows: troponin at $24 \mathrm{~h}$ after surgery, lactate at $0 \mathrm{~h}, 6 \mathrm{~h}, 12 \mathrm{~h}, 24 \mathrm{~h}$, and maximal lactate after surgery, and maximal creatinine, aspartate aminotransferase (AST), and alanine aminotransferase (ALT) levels measured during the hospitalization period. The preoperatively measured parameters were Aristotle score [5], invasive or noninvasive ventilator support, oxygen saturation levels, and whether the surgery was elective or nonelective. The surgical parameters were the cardiopulmonary bypass (CPB) and aortic crossclamp (ACC) times. The postoperative parameters were invasive and noninvasive ventilator support, length of ventilation, length of hospitalization ( $\mathrm{LOH}$ ), need for reintubation, need for extracorporeal membrane oxygenation (ECMO), maximal inotropic support, and number of days during which inotropic support was given. These parameters were divided into two groups in order to examine correlations. The first group contained the parameters that were related to the $\mathrm{LOH}$ (i.e., invasive and noninvasive ventilation days and the total number of hospitalization days), and they did not account for children who died following surgery. The second group contained all the other parameters and accounted for all the children in the study.

The appearance of cardiac and inflammatory complications was the primary outcome. Cardiac complications were defined as the need for cardiorespiratory resuscitation, pericardial and/or pleural effusion requiring treatment or drainage, and postoperative arrhythmia (junctional ectopic, supraventricular, and ventricular tachycardias). Inflammatory complications were defined as the need for antibiotic treatment. Twenty-six patients comprised the group that sustained complications, of whom 19 patients were diagnosed with complications that involved the heart. Laboratory parameters, LOH, and higher or lower than the median maximal lactate value were the secondary outcomes.

\section{Sample Preparation, RNA Extraction, and CDNA Preparation and Quantification}

All samples were processed within $24 \mathrm{~h}$ of surgery. The blood was centrifuged at $4{ }^{\circ} \mathrm{C}$ for $10 \mathrm{~min}$ at $1200 \mathrm{~g}$ followed by separation of serum. Centrifugation was then repeated at 4 ${ }^{\circ} \mathrm{C}$ for $10 \mathrm{~min}$ at $10000 \mathrm{~g}$. RNA was extracted from $250 \mu \mathrm{l}$ serum to which $5.6 \times 10^{8}$ copies of cel-miRNA39 were added using TRI-Reagent ${ }^{\circledR}-\mathrm{LS}$ (Sigma). RNA was resuspended in $45 \mu \mathrm{l} \mathrm{H}_{2} \mathrm{O}$ of which $5 \mu \mathrm{l}$ was taken for cDNA synthesis (TaqMan® MicroRNA-RT-Kit, ABI, \#4366597) using a specific primer. MiRNA quantification was performed on a StepOne ${ }^{\mathrm{TM}}$ PCR (QPCR). Reactions $(10 \mu \mathrm{l}$ total) were run in triplicates and consisted of: $5 \mu$ PCR-mix (ABI, \#AB4444557), $0.5 \mu \mathrm{l}$ of either of the Taqman miRNA assays (ABI, \#AB-4427975): cel-miRNA39 (\#000200) or miRNA208a (\#000511), and $1.5 \mu \mathrm{l}$ of the cDNA and $3 \mu \mathrm{l} \mathrm{H}_{2} \mathrm{O}$. The cycle threshold $(\mathrm{Ct})$ values were calculated with the StepOne software v2.3. $\Delta \mathrm{Ct}$ values are calculated by subtracting the $\mathrm{Ct}$ of miRNA-208a from the Ct of cel-miRNA39 of the same sample. The $\Delta \mathrm{Ct}$ values are inversely correlated with the amount of template miRNA present in the reaction. The RQ was analyzed using the $\Delta \Delta \mathrm{Ct}$ method.
Table 1 Characteristics of the study population

\begin{tabular}{llll}
\hline Characteristic & All patients $(n=79)$ & $\begin{array}{l}\text { Patients with oxygen } \\
\text { saturation levels below } \\
90 \%(n=38)\end{array}$ & $\begin{array}{l}\text { Patients with oxygen } \\
\text { saturation levels above } \\
90 \%(n=41)\end{array}$ \\
\hline Age (months) & $6.8(0.4-33.9)$ & $3.6(0.2-17.0)$ & $11.76(1.2-42)$ \\
Weight $(\mathrm{kg})$ & $6(3.3-12)$ & $4.3(3.1-8.6)$ & $6.7(3.9-14.6)$ \\
Operations $(n)$ & $1(1-2)$ & $1(1-3)$ & $1(1-2)$ \\
\hline
\end{tabular}

Mean values are reported with the interquartile range in parentheses 
Table 2 The surgical procedures

\begin{tabular}{lll}
\hline Type of operation & Number of patients & Percentage of patients $(\%)$ \\
\hline VSD/ASD & 17 & 22 \\
AV canal & 4 & 5 \\
TGA & 13 & 17 \\
TOF & 9 & 11 \\
COA + arch repair & 7 & 9 \\
BT shunt & 5 & 6 \\
Norwood & 5 & 6 \\
Glenn & 5 & 6 \\
Fontan & 6 & 8 \\
RV to PA conduit & 8 & 10 \\
Total & 79 & 100 \\
\hline
\end{tabular}

$V S D$ ventricular septal defect, $A S D$ atrial septal defect, $A V$ atrioventricular, $T G A$ transposition of the great arteries, TOF tetralogy of Fallot, COA coarctation of the aorta, $B T$ Blalock Taussig, $R V$ right ventricle, $P A$ pulmonary artery

\section{Statistical Analysis}

Categorical variables were described as frequency and percentage. Continuous variables were evaluated for normal distribution using histograms and Q-Q plots. The normally distributed continuous variable was described as mean and standard deviation (SD), and non-normally distributed continuous variables as median and interquartile range (IQR). Continuous variables were compared between patients with and without complications using an independent sample $t$ test. Correlations between continuous variables were evaluated using the Spearman correlation coefficient. Univariate and multivariate logistic regressions were used to evaluate the association between the miRNA expression and the complications. The multivariate models were performed twice. The model included age and Aristotle as confounders. The area under the receiver operating characteristic (ROC) curve was used to evaluate the discrimination ability of the miRNA. Odds ratios (OR) with $95 \%$ confidence intervals $(\mathrm{CI})$ were reported. All statistical tests were 2-tailed, and $p<0.05$ was considered statistically significant. All calculations were performed using SPSS (Ver. 25.0). All statistical analyses were performed on three groups of patients: (1) the entire cohort, (2) patients with oxygen saturations below $90 \%$, and (3) patients with oxygen saturations above $90 \%$.

The protocol of this study was approved by the Institutional Review Board of the Chaim Sheba Medical Center. Informed consent was obtained from the legal representatives of all subjects.

\section{Results}

Seventy-nine consecutive children who underwent cardiac surgery for repair of congenital anomalies at Safra Children's Hospital between 2012 and 2016 were enrolled. There were 34 (43\%) females and $45(57 \%)$ males. The characteristics of the population are shown in Table 1 and the surgical procedures are listed in Table 2. The surgical characteristics, the laboratory parameters, and the preoperative and postoperative characteristics of the study patients are summarized in Tables 3, 4, and 5 .
Table 3 Surgical characteristics and laboratory parameters

\begin{tabular}{llll}
\hline Parameter & All patients $(n=79)$ & $\begin{array}{l}\text { Patients with oxygen } \\
\text { saturation levels } \\
\text { below } 90 \%(n=38)\end{array}$ & $\begin{array}{l}\text { Patients with oxygen } \\
\text { saturation levels } \\
\text { above 90\% }(n=41)\end{array}$ \\
\hline CPB time (min) & $62(30-100)$ & $79(39-109.5)$ & $50(29-96.5)$ \\
ACC time (min) & $30(0-70)$ & $38.5(0-76.2)$ & $30(0-60.5)$ \\
Lactate 0 h (mg/dL) & $12(9-17)$ & $13.5(8.7-20.5)$ & $12(8.5-14.5)$ \\
Lactate 6 h (mg/dL) & $20(14-34)$ & $26.5(16.7-52)$ & $18(11.5-30)$ \\
Lactate 12 h (mg/dL) & $15(12-23)$ & $18(14-33.5)$ & $12(10-19)$ \\
Lactate 24 h (mg/dL) & $14(11-21)$ & $19(13.25-26.5)$ & $12(9.5-14.5)$ \\
Max. postop. lactate (mg/dL) & $42(26-79)$ & $46(29.2-87.7)$ & $33(24-58.5)$ \\
Troponin $(\mathrm{ng} / \mathrm{mL})$ & $11.4(4.68-25)$ & $11.6(5.2-35.1)$ & $10(4-23.9)$ \\
Max creatinine (mg/dL) & $0.58(0.44-0.74)$ & $0.6(0.5-0.8)$ & $0.5(0.3-0.6)$ \\
Max ALT U/L & $25(19-36)$ & $25(18-47.7)$ & $25(21-32.5)$ \\
Max. AST U/L & $115(85-187)$ & $122(85-306.5)$ & $1113(75.5-169)$ \\
Preop. saturation & $91(84-97)$ & $83.5(79-86)$ & $97(96-99)$ \\
Max. inotropic score & $14.9(5-22.5)$ & $15(5-27.2)$ & $10(6-18)$ \\
\hline
\end{tabular}

Mean values are reported with the interquartile range in parentheses

$C P B$ cardiopulmonary bypass, $A C C$ aortic cross clamp, Max. maximal, postop. postoperative, preop. preoperative, $A L T$ alanine aminotransferase, $A S T$ aspartate aminotransferase 
Table 4 Characteristics during hospitalization

\begin{tabular}{llll}
\hline Characteristic (days) & $\begin{array}{l}\text { All patients } \\
(n=79)\end{array}$ & $\begin{array}{l}\text { Patients with oxygen } \\
\text { saturation levels } \\
\text { below } 90 \%(n=38)\end{array}$ & $\begin{array}{l}\text { Patients with oxygen } \\
\text { saturation levels } \\
\text { above 90\% }(n=41)\end{array}$ \\
\hline LOH & $9(6-14)$ & $11(7-14)$ & $7(4-10)$ \\
Ventilated noninvasively & $1.5(0.34-4.28)$ & $3.4(1-5.4)$ & $0.6(0.1-2.2)$ \\
Ventilated invasively & $0.91(0.22-2.06)$ & $1(0.3-2.3)$ & $0.5(0.1-1.6)$ \\
Inotropic support & $2(1-4)$ & $2(1-4)$ & $1.5(0.8-3)$ \\
Respiration before operation & $0(0-0.75)$ & $0(0-2)$ & $0(0-0)$ \\
\hline
\end{tabular}

Mean values are reported with the interquartile range in parentheses

$\mathrm{LOH}$ length of hospitalization

\section{Preoperative Levels of miRNA-208a in the Entire Cohort}

The expression of miRNA-208a was measured immediately before the beginning of the operation in all the patients. The patients were divided into two groups: those that did not sustain postoperative complications and those that did. The relative levels of expression of miRNA-208a were 3.7 times higher in the group of children with an uncomplicated postoperative course $(p=0.03)$ (Fig. 1).

Logistic regression analysis showed an association between the preoperative levels of miRNA208a and the risk of developing complications: the lower the level of miRNA208a in the patient's blood before the operation, the higher was the risk for developing complications following surgery (crude OR 1.16; 95\% CI 1.01-1.33; $p=0.03$ ). This association remained significant after adjusting for the child's age and Aristotle score (adjusted OR 1.14; 95\% CI 0.99-1.32; $p=$ 0.05) (Table 6).

Preoperative levels of miRNA-208a were studied as predictors of postoperative complications using an ROC curve and the area under the curve (AUC). The result of this analysis reached a level of significance, with an AUC of $64 \%$ (95\% CI 51.1-77.0\%; $p=0.04$ ) (Fig. 2).

There was a significant $(p=0.03)$ inverse correlation between the amount of miRNA-208a before surgery and the Aristotle score. There was also a correlation $(p=0.01)$ between preoperative low oxygen saturation values with a shorter LOH.

\section{Preoperative Levels of miRNA-208a in the Patients with Low Oxygen Saturation}

We hypothesized that miRNA-208a may have better prediction performances in a more homogenous group of patients in relation to their oxygen saturation levels. To test that hypothesis, we divided our cohort into two groups: patients with oxygen saturation levels below 90\% (hypoxic) and patients with oxygen saturation levels above $90 \%$ (normoxic). We analyzed the data on the preoperative levels of miRNA-208a in each group separately, looking for correlations between the amount of miRNA-208a and postoperative outcome. The relative levels of expression of miRNA-208a before surgery among the children with oxygen saturation levels below $90 \%(n=38)$ were 9.0 times higher for those without postoperative cardiac complications compared with the children who did sustain them ( $p=0.01$ ) (Fig. 3). Logistic regression calculations in the group of patients with oxygen saturation levels below $90 \%$ yielded a statistically significant association between the preoperative level of miRNA-208a and the risk of developing postoperative complications: the lower the level of miRNA-208a in the patient's blood before the operation, the higher was the risk for developing cardiac complications following surgery (crude OR 1.28; 95\% CI 1.02-1.60; $p=0.02$ ).

Table 5 Categorical characteristics

\begin{tabular}{llll}
\hline Characteristic & All patients $(n=79)$ & $\begin{array}{l}\text { Patients with oxygen } \\
\text { saturation levels } \\
\text { below } 90 \%(n=38)\end{array}$ & $\begin{array}{l}\text { Patients with oxygen } \\
\text { saturation levels } \\
\text { above } 90 \%(n=41)\end{array}$ \\
\hline Reintubation/no reintubation & $16 / 59(27 \%)$ & $8 / 30(21 \%)$ & $8 / 33(19.5 \%)$ \\
ECMO/no ECMO & $3 / 76(4 \%)$ & $3 / 35(7.8 \%)$ & $0 / 41(0 \%)$ \\
Nonelective/elective surgery & $27 / 52(34 \%)$ & $19 / 19(50 \%)$ & $8 / 33(19.5)$ \\
complication & $19 / 4 / 56(24 / 5 / 71 \%)$ & $13 / 3 / 22(34.2 / 7.8 / 57.8 \%)$ & $9 / 1 / 31(21.9 / 2.4 / 75.6 \%)$ \\
\hline
\end{tabular}

ECMO extracorporeal membrane oxygenation 


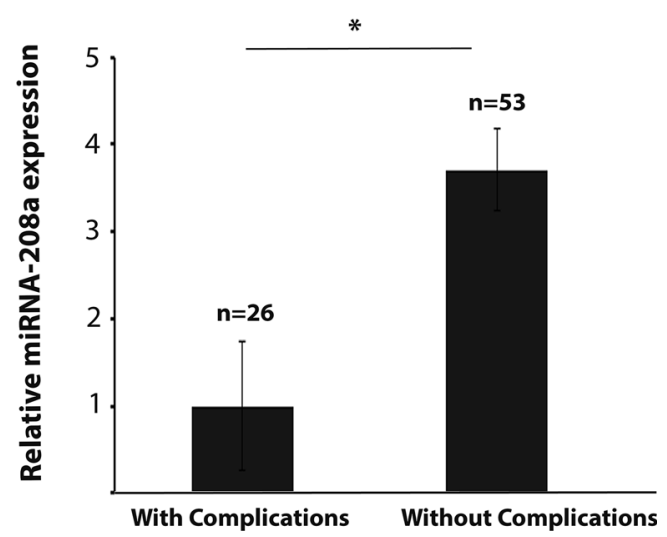

Fig. 1 Relative average miRNA-208 expression in all the patients. Expression levels were quantified by QPCR and the average level of the group of patients that sustained complications was set as 1 . Values are shown \pm SEM

This association remained significant after adjusting for the child's age and Aristotle score (adjusted OR 1.27; 95\% CI $1.01-1.59 ; p=0.03$ ) (Table 6).

The ability of preoperative levels of miRNA-208a to predict postoperative cardiac complications in the group of patients with oxygen saturation levels below $90 \%$ was studied using an ROC curve and the AUC. This analysis reached a level of significance, with an AUC of $71 \%$ (95\% CI 54.5$88.1 \%$; $p=0.02$ ) (Fig. 4). A $\Delta$ Ct of 18.0 can serve as a cutoff value for the prediction of the risk of complications following heart surgery in that group of patients. Specifically, $\Delta \mathrm{Ct}>18.0$ predicted that the child will sustain complications whereas $\Delta \mathrm{Ct}<18.0$ predicted that he/she will not. The sensitivity of this value was $81 \%$ and its specificity was $46 \%$.

\section{Preoperative Levels of miRNA-208a in the Patients with High Oxygen Saturation Levels}

The 41 children who had oxygen saturation levels above $90 \%$ had significant inverse correlations between the amount of miRNA-208a before the operation and the following postoperative parameters: fewer days of postoperative invasive or noninvasive ventilation $(p=0.05)$ and fewer days of inotropic support $(p=0.00)$. In addition, higher levels of miRNA-208a correlated with lower lactate values $(p=0.01)$, with lower creatinine levels $(p=0.00)$, and with lower Aristotle scores $(p=0.00)$. These results also indicated that higher levels of preoperative miRNA-208a correlated with a better outcome in this group (Table 7).

Dividing the patients with oxygen saturation levels above $90 \%$ according to their postoperative lactate values revealed a significant $(p=0.01)$ difference in their preoperative miRNA208a levels (Fig. 5). Patients with higher postoperative lactate values had lower preoperative miRNA-208a levels. This result supports the ability of preoperative miRNA-208a levels to predict the patient's postoperative outcome. It is also worth noting that dividing this group of patients according to their $\mathrm{LOH}$ (more or less than the median of 8 days) yielded a substantial, although not significant, difference in the amount of preoperative miRNA-208a: specifically, children with a shorter LOH had 2.7 times more miRNA-208a in serum before their operation than those with longer LOHs.

\section{Discussion}

The findings of our current investigation revealed that preoperative levels of circulating cardiac miRNA-208a in the serum are predictive of the pediatric patient's complications following surgery for life-threatening anatomical malformations associated with CHD. To the best of our knowledge, this is the first study to identify a miRNA which can serve as a single preoperative biomarker effective in predicting the postoperative outcome in this setting. This is a continuation of our previous study in which we identified that circulating miRNA-208a can serve as an accurate biomarker for predicting the risk of developing postoperative complications $6 \mathrm{~h}$ following surgery: high levels of miRNA-208a in the serum several hours after surgery were indicative of complications which increased the risk of sustaining a worse outcome [4].

The potential benefit of being able to preoperatively identify patients at high risk for complications following heart surgery for such life-threatening conditions is enormous. Accurate preoperative risk assessment at an individual patient level enhances clinical decision-making of postoperative patient management and the estimation of associated risks. To

Table 6 Odds ratio

\begin{tabular}{llllr}
\hline Group & Adjustment & $\begin{array}{l}\text { With complications } \\
n(\%)\end{array}$ & $\begin{array}{l}\text { Without complications } \\
n(\%)\end{array}$ & OR (95\% CI) \\
\hline All patients & No & $26(33.3)$ & $52(66.7)$ & $1.16(1.01-1.33)$ \\
All patients & Age, Aristotle & $26(33.3)$ & $52(66.7)$ & 0.03 \\
Patients with low oxygen saturation & No & $16(42.1)$ & $22(57.9)$ & 0.05 \\
Patients with low oxygen saturation & Age, Aristotle & $16(42.1)$ & $22(57.9)$ & $0.09-1.32)$ \\
\hline
\end{tabular}




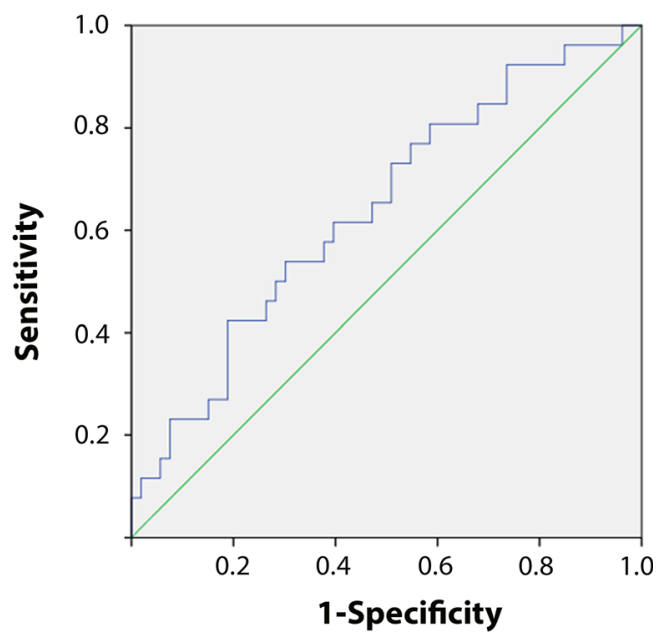

Fig. 2 ROC curve showing the predictive power of preoperative levels of miRNA-208a to predict postoperative complications for all the patients

date, preoperative prediction of the postoperative course involves the use of the Aristotle score which takes into account the complexity of the surgical procedure and predicts 30-day mortality and LOH during the first postoperative week. In addition, recent papers have shown the potential benefit of preoperative ST2 levels to identify children with increased risk of mortality or readmission after pediatric heart surgery $[6,7]$. We were intrigued by the possibility that miRNA-208a could also serve as a preoperative biomarker for the child's potential sequelae. Our results indeed showed that high expression levels of preoperative circulating miRNA-208a were predictive of a better postoperative outcome, as reflected by fewer complications following surgery and, accordingly, were correlated with a low Aristotle score and a shorter LOH.

We hypothesized that the elevated miRNA-208a found in the sera of patients before the operation was secondary to the presence of a disturbance in normal physiology and that such patients benefited from preconditioning of the heart, thereby

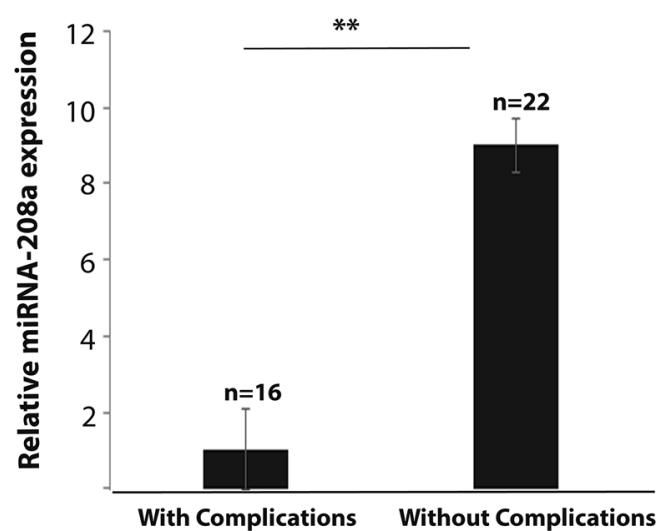

Fig. 3 Relative average miRNA-208 expression in children with oxygen saturation below 90\%. Expression levels were quantified by QPCR and the average level of the group of patients that sustained complications was set as 1 . Values are shown \pm SEM

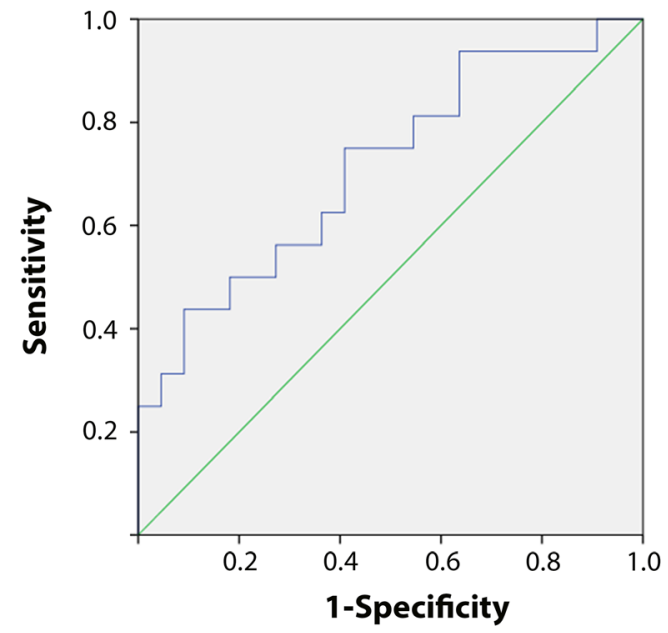

Fig. 4 ROC curve showing the predictive power of preoperative levels of miRNA-208a to predict postoperative complications for the patients with an oxygen saturation level below $90 \%$

leading to a greater likelihood of an uncomplicated postoperative course. The hearts of those patients were "prepared" before surgery by a yet to be determined mechanism, which enabled them to cope optimally with the stress caused during and immediately after the operation, with the result of fewer postoperative complications. Our study is not the first to suggest that a beneficial postoperative outcome is seen in patients whose hearts were exposed to preoperative stress. Remote ischemic preconditioning (RIPC) was found to be clinically effective in a study by $\mathrm{Wu}$ et al. on TOF children undergoing open heart surgery [8]. Those authors showed that RIPC attenuated myocardial ischemia/reperfusion (I/R) injury and improved the short-term prognosis of those patients [8]. Not surprisingly, miRNAs have been reported to mediate such protection of the heart in several studies $[9,10]$. The involvement of miRNAs in protective mechanisms has also been described in preconditioning of the brain [11].

The source of the high levels of circulating miRNA-208a before surgery has yet to be determined. In general, extracellular circulating miRNAs originate from two main sources: they can be merely byproducts of cellular activity and cell death [12], or they can be the result of a selective export system which enables the secreted miRNA to be transferred to a recipient cell where it can carry out its role [13]. We and others have shown that the presence of miRNA-208a in the blood correlates with damage caused to the heart $[4,14,15]$; however, there are no studies that define the exact mechanism of the export system. Notably, however, circulating miRNA208a in the blood could be a trigger for inducing a heartprotective mechanism whose results become evident following surgery in both of the above-mentioned scenarios. A role for miRNA-208a in communicating signals from the heart to other parts of the body has been reported by Feng et al., who found that cardiac miRNA-208a released into the circulation following myocardial $\mathrm{I} / \mathrm{R}$ is capable of activating innate 
Table 7 Correlations with postoperative parameters

\begin{tabular}{|c|c|c|c|c|c|}
\hline & $\begin{array}{l}\text { Ventilation } \\
\text { (days) }\end{array}$ & $\begin{array}{l}\text { Inotropic_support } \\
\text { (days) }\end{array}$ & $\begin{array}{l}\text { Blood } \\
\text { lactate } \\
(\max )\end{array}$ & $\begin{array}{l}\text { Creatinine } \\
(\max )\end{array}$ & Aristotle \\
\hline Correlation Coefficient & 0.30 & 0.42 & 0.37 & 0.49 & 0.44 \\
\hline Sig. (2-tailed) & 0.05 & 0.00 & 0.01 & 0.00 & 0.00 \\
\hline$N$ & 40 & 37 & 41 & 40 & 40 \\
\hline
\end{tabular}

Correlations were calculated for $\Delta \mathrm{Ct}$ values

immunity [16]. We suggest that because the hearts of the children that were subjected to prolonged physiological stress until the date of the operation had the opportunity to be more prepared, and possibly more protected, from the effects of the operation, those children sustained fewer postoperative complications. Although the correlation between the levels of miRNA-208a and the postoperative course was more significant in the cyanotic patients, results pointing in the same direction were also obtained for the non-cyanotic ones. This observation strengthens our position that exposing the heart to abnormal physiological constraints, as could be expected in children with low oxygen saturation, produces a stronger "signal" to provoke a protective mechanism, whatever it may be.

This is a pilot study which is intended to serve as the basis for a larger investigation designed to examine the value of obtaining preoperative miRNA levels for the purpose of guiding decisions regarding surgery and the postoperative management that will need to be provided for a specific patient. In addition to demonstrating the benefit of having such information, our results suggest that miRNA-208a may serve as a preoperative target for therapy in children with CHD. Increasing its levels in the blood of such patients may trigger the preconditioning of their heart and thus contribute to a smoother postoperative course.

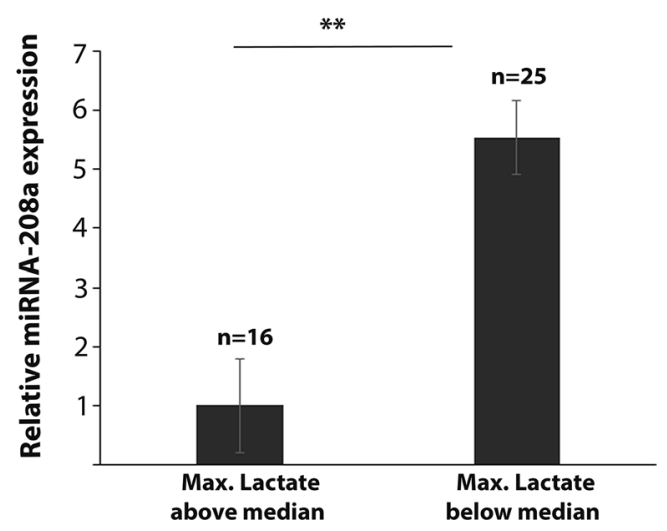

Fig. 5 Relative average miRNA-208 expression in children with oxygen saturation more than $90 \%$. Expression levels were quantified by QPCR and the average level of the group of patients for whom lactate maximal levels were above the median (i.e., $>42 \mathrm{mg} / \mathrm{dL}$ ) was set as 1 . Values are shown \pm SEM
We are aware of a main limitation of our study that bears mention. Our cohort comprises patients with diverse CHDs which necessarily affect their medical parameters. Our attempts to divide the cohort into homogenous groups resulted in groups too small to enable us to reach statistically significant results. We are also aware that our small sample size may be a limitation for our multivariate analysis results.

\section{Conclusions}

We have shown that the level of preoperative miRNA-208a in serum is a reliable biomarker for the prediction of patients who are at risk to develop postoperative complications. Unlike other extracellular RNA molecules, extracellular miRNAs are remarkably stable in serum and, together with their tissue specificity and the ease by which they can be detected and quantified, they have sparked great interest in their use as clinical biomarkers for a wide range of medical states. Such a biomarker can help in surgical comorbidity assessment, which is an integral part of patient risk stratification. Further studies to assess its applicability to specific cardiac surgeries and to other invasive cardiac procedures should be performed in a larger group of patients.

Acknowledgments We thank Amisragas for their continuous support of the Department of Intensive Care.

Funding Information This work was funded with institutional departmental funds.

\section{Compliance with Ethical Standards}

All procedures performed in this study involving human participants were in accordance with the ethical standards of the institutional research committee and with the 1964 Helsinki declaration and its later amendments. Ethical approval was obtained from the Ethics Committee of the Sheba Medical Center. No animal studies were carried out by the authors for this article.

Conflict of Interest The authors declare that they have no conflict of interest.

Informed Consent Informed consent was obtained from all individual participants or their parents/legal guardians included in the study. 


\section{References}

1. Gaies, M., Pasquali, S. K., Donohue, J. E., Dimick, J. B., Limbach, S., Burnham, N., Ravishankar, C., Ohye, R. G., Gaynor, J. W., \& Mascio, C. E. (2016). Seminal postoperative complications and mode of death after pediatric cardiac surgical procedures. The Annals of Thoracic Surgery, 102, 628-635.

2. Biccard, B. M., \& Rodseth, R. N. (2011). Utility of clinical risk predictors for preoperative cardiovascular risk prediction. British Journal of Anaesthesia, 107, 133-143.

3. Pogribny, I. P. (2018). MicroRNAs as biomarkers for clinical studies. Experimental Biology and Medicine, 243, 283-290.

4. Zloto, K., Tirosh-Wagner, T., Bolkier, Y., Bar-Yosef, O., Vardi, A., Mishali, D., Nevo-Caspi, Y., \& Paret, G. (2018). MiRNA-208a as a sensitive early biomarker for the postoperative course following congenital heart defect surgery. Pediatric Cardiology, 39, 15651571.

5. Bojan, M., Gerelli, S., Gioanni, S., Pouard, P., \& Vouhé, P. (2011). The Aristotle comprehensive complexity score predicts mortality and morbidity after congenital heart surgery. The Annals of Thoracic Surgery, 91, 1241-1221.

6. Parker, D. M., Everett, A. D., Stabler, M. E., Vricella, L., Jacobs, M. L., Jacobs, J. P., Thiessen-Philbrook, H., Parikh, C. R., \& Brown, J. R. (2019). Biomarkers associated with 30-day readmission and mortality after pediatric congenital heart surgery. Journal of Cardiac Surgery, 34, 329-336.

7. Parker, D. M., Everett, A. D., Stabler, M. E., Vricella, L., Jacobs, M. L., Jacobs, J. P., Parikh, C. R., Pasquali, S. K., \& Brown, J. R. (2019). Novel biomarkers improves prediction of 365-day readmission after pediatric congenital heart surgery. The Annals of Thoracic Surgery.

8. Wu, Q., Wang, T., Chen, S., Zhou, Q., Li, H., Hu, N., Feng, Y., Dong, N., Yao, S., \& Xia, Z. (2018). Cardiac protective effects of remote ischaemic preconditioning in children undergoing tetralogy of fallot repair surgery: a randomized controlled trial. European Heart Journal, 39, 1028-1037.
9. Wang, X., Ha, T., Zou, J., Ren, D., Liu, L., Zhang, X., Kalbfleisch, J., Gao, X., Williams, D., \& Li, C. (2014). MicroRNA-125b protects against myocardial ischaemia/reperfusion injury via targeting p53-mediated apoptotic signaling and TRAF6. Cardiovascular Research, 102, 385-395.

10. Lusha, E., Hong, J., \& Zhibing, L. (2018). MicroRNA-144 attenuates cardiac ischemia/reperfusion injury by targeting FOXO1. Experimental and Therapeutic Medicine, 17, 2152-2160.

11. Jimenez-Mateos, E. M. (2015). Role of microRNAs in innate neuroprotection mechanisms due to preconditioning of the brain. Frontiers in Neuroscience, 9, 118.

12. Xin, Y., Yang, C., \& Han, Z. (2016). Circulating miR-499 as a potential biomarker for acute myocardial infarction. Annals of Translational Medicine, 4, 135.

13. Zernecke, A., Bidzhekov, K., Noels, H., Shagdarsuren, E., Gan, L., Denecke, B., Hristov, M., Köppel, T., Jahantigh, M. N., Lutgens, E., Wang, S., Olson, E. N., Schober, A., \& Weber, C. (2009). Delivery of microRNA-126 by apoptotic bodies induces CXCL12dependent vascular protection. Science Signaling, 2, ra81.

14. Liu, H., Yang, N., Fei, Z., Qiu, J., Ma, D., Liu, X., Cai, G., \& Li, S. (2016). Analysis of plasma miR-208a and miR-370 expression levels for early diagnosis of coronary artery disease. Biomedical Reports, 5, 332-336.

15. Bi, S., Wang, C., Jin, Y., Lv, Z., Xing, X., \& Lu, Q. (2015). Correlation between serum exosome derived miR-208a and acute coronary syndrome. International Journal of Clinical and Experimental Medicine, 8, 4275-4280.

16. Feng, Y., Zou, L., Yan, D., Chen, H., Xu, G., Jian, W., Cui, P., \& Chao, W. (2017). Extracellular microRNAs induce potent innate immune responses via TLR7/MyD88-dependent mechanisms. Journal of Immunology, 199, 2106-2117.

Publisher's Note Springer Nature remains neutral with regard to jurisdictional claims in published maps and institutional affiliations. 